\title{
Quantum coherence and population trapping in three-photon processes
}

\author{
Caroline Champenois* \\ Physique des Interactions Ioniques et Moléculaires (CNRS UMR 6633), Université de Provence, \\ Centre de Saint Jérôme, Case C21, 13397 Marseille Cedex 20, France \\ Giovanna Morigi \\ Grup d'Optica, Departament de Fisica, Universitat Autonoma de Barcelona, 08193 Bellaterra, Spain
}

\author{
Jürgen Eschner \\ ICFO_Institut de Ciències Fotòniques, Mediterranean Technology Park, 08860 Castelldefels, Barcelona, Spain \\ (Received 30 July 2006; published 3 November 2006)
}

\begin{abstract}
The spectroscopic properties of a single tightly trapped atom are studied, when the electronic levels are coupled by three laser fields in an $N$-shaped configuration of levels, whereby a $\Lambda$-type level system is weakly coupled to a metastable state. We show that depending on the laser frequencies the response can be tuned from coherent population trapping at two-photon resonance to a behavior at three photon resonance, where the metastable state can get almost unit occupation in a wide range of parameters. For certain parameter regimes the system switches spontaneously between dissipative and coherent dynamics over long time scales.
\end{abstract}

DOI: 10.1103/PhysRevA.74.053404

PACS number(s): 32.80.Pj, 42.50.Gy, 42.50.Lc.

\section{INTRODUCTION}

Atomic coherence has been demonstrated to be an efficient tool for achieving control of the interaction between electromagnetic fields and an atomic sample. It is at the basis, for instance, of the realization of quantum nonlinear optical devices [1-3], quantum phase gates [4-6], atomic transistors [7], and high-precision measurement techniques [8].

A paradigmatic system exhibiting atomic coherence effects is the so-called $\Lambda$ configuration of atomic levels, where two (meta)stable states are coupled by two light fields to the same excited state. When the coupling happens at twophoton resonance, the system exhibits coherent population trapping, by forming an atomic coherence between the two stable states which decouples from the radiation [9]. More complex configurations of levels offer richer dynamics, whose understanding is relevant for applications of coherent control of complex systems [10-12]. The interpretation of their dynamics is often nontrivial, yet in some parameter regimes analogies may be found with simpler level systems which are better understood. This usually helps developing tools for controlling and manipulating the quantum dynamics of the more complex system through external parameters $[10,13,14]$.

In this work we study how the dynamics of a $\Lambda$ system is modified by an additional coupling of one of the stable states to a fourth, metastable state, as depicted in Fig. 1. Due to its shape, we denote this configuration as $N$-level scheme. We show that even weak coupling to the fourth level gives rise to strong modification of the dynamics whenever the threephoton resonance condition between the outer levels is fulfilled. In particular, in certain parameter regimes the fourth state exhibits quasi-unit occupation probability; in other situations the behavior switches from dissipative transient to coherent asymptotic dynamics.

\footnotetext{
*Electronic address: caroline.champenois@up.univ-mrs.fr
}

Our analysis applies to alkalilike atomic species, as well as to some alkaline-earth elements. We consider in particular the case of a single ${ }^{40} \mathrm{Ca}^{+}$ion in a radiofrequency trap. For this system we also study the effect of the oscillatory motion on the dynamical behavior.

$N$-level schemes have been extensively studied in Refs. [15-17] in the framework of electromagnetically induced absorption [18]. A peculiar difference of those systems with the scheme we investigate here is the stability of the fourth level, which critically affects the response of the system. As a result, the narrow lines at three-photon resonance, which we report in this manuscript, cannot be explained in terms of transfer of coherence $[15,16]$, but are instead intimately related to coherent population trapping, as we will argue. Coherent population trapping and dark resonances have been discussed in Refs. $[19,20]$ for a configuration similar to the one we discuss here, with the important difference that in Refs. $[19,20]$ the unstable state can decay in all three stable states. In this latter system, Doppler-free-like absorption resonances [21] and three-photon electromagnetically induced transparency (EIT) [22] have been observed. We will comment on how our model system reproduces and differs from these studies. Finally, three-photon resonances have been studied in connection with metrology [23,24]. Indeed, the type of system we consider is encountered in atomic clocks, where transition $|S\rangle \rightarrow|Q\rangle$ is the clock transition of, say, a ${ }^{40} \mathrm{Ca}^{+}$ion or a ${ }^{87} \mathrm{Sr}$ atom, and the dynamics we predict may have applications for high-precision optical clocks. In this context, we also study how the oscillatory motion inside an ion trap modifies the spectroscopic signals.

This article is organized as follows. In Sec. II the theoretical model is introduced, in Sec. III the theoretical analysis and predictions are reported, and in Sec. IV we extend it when the oscillatory motion of a trapped atom is considered. In Sec. V discussions and conclusions are reported, and in the appendix the model at the basis of calculations in Sec. IV is described. 


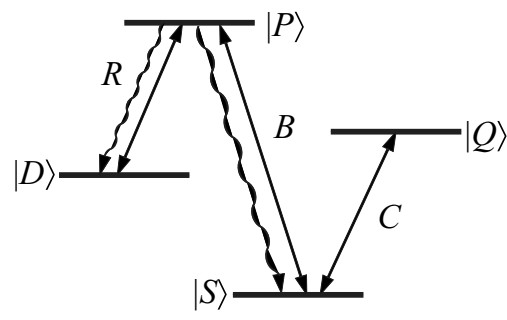

FIG. 1. $N$ Level scheme. The states $|D\rangle,|P\rangle,|S\rangle$ form a $\Lambda$ configuration; additionally, state $|S\rangle$ couples weakly to the metastable state $|Q\rangle$. The solid lines and letters $R, B, C$ indicate the laser couplings, the wavy lines the radiative decay channels. Parameters and possible atomic species are discussed in the text.

\section{THEORETICAL MODEL}

The atomic system we consider is composed by four electronic levels which are coupled by laser fields, according to the $N$-shaped scheme depicted in Fig. 1. Here, states $|S\rangle,|D\rangle$, and $|Q\rangle$ are (meta)stable, state $|P\rangle$ is unstable and decays radiatively into $|S\rangle$ and $|D\rangle$. This configuration of levels is realized, for instance, in alkaline-earth atoms with hyperfine structure and in alkalilike ions with a metastable $d$ orbital, such as $\mathrm{Hg}^{+}, \mathrm{Ba}^{+}, \mathrm{Sr}^{+}$, or $\mathrm{Ca}^{+}$. In this manuscript we make reference to the typical parameters of ${ }^{40} \mathrm{Ca}^{+}$ion. In this case, the relevant levels can be identified with the states $|S\rangle=\left|S_{1 / 2}\right\rangle,|P\rangle=\left|P_{1 / 2}\right\rangle,|D\rangle=\left|D_{3 / 2}\right\rangle$, and $|Q\rangle=\left|D_{5 / 2}\right\rangle$. Here, the transition $|S\rangle \rightarrow|Q\rangle$, marked by $C$ in Fig. 1, is an electric quadrupole transition with a linewidth of the order of $0.1 \mathrm{~Hz}$, while $|S\rangle$ and $|D\rangle$ couple to the excited state $|P\rangle$ with electric dipole transitions (marked by $B$ and $R$, respectively). We denote by $\omega_{i j}(i, j=S, P, D, Q)$ the resonance frequencies of the transitions. Transitions $|S\rangle \rightarrow|P\rangle$ and $|D\rangle \rightarrow|P\rangle$ are coupled by lasers at frequency $\omega_{B}$ and $\omega_{R}$ and Rabi frequencies $\Omega_{B}$ and $\Omega_{R}$, respectively. These three states form a $\Lambda$-configuration of levels. Transition $|S\rangle \rightarrow|Q\rangle$ is driven by a laser at frequency $\omega_{C}$ and Rabi frequency $\Omega_{C}$.

We denote by $\rho$ the density matrix for the ion's internal degrees of freedom, while we treat the center of mass variables classically. We denote by $x(t)$ the time-dependent position of the atom. The master equation for the density matrix $\rho$ is

$$
\frac{\partial}{\partial t} \rho=-\frac{i}{\hbar}[H, \rho]+\mathcal{L} \rho,
$$

where Hamiltonian $H$ gives the coherent dynamics and is decomposed into the terms

$$
H=H_{0}+H_{I},
$$

where

$$
H_{0}=-\hbar \Delta_{B}|P\rangle\left\langle P\left|-\hbar \Delta_{C}\right| Q\right\rangle\left\langle Q\left|+\hbar\left(\Delta_{R}-\Delta_{B}\right)\right| D\right\rangle\langle D|
$$

gives the internal energies in the reference frames of the lasers, with detunings defined as $\Delta_{B}=\omega_{B}-\omega_{P S}, \Delta_{R}=\omega_{R}$ $-\omega_{P D}$, and $\Delta_{C}=\omega_{C}-\omega_{Q S}$, while

$$
\begin{aligned}
H_{I}= & \frac{\hbar \Omega_{B}}{2} e^{i k_{B} x(t)}|P\rangle\left\langle S\left|+\frac{\hbar \Omega_{R}}{2} e^{i k_{R} x(t)}\right| P\right\rangle\langle D| \\
& +\frac{\hbar \Omega_{C}}{2} e^{i k_{C} x(t)}|Q\rangle\langle S|+\text { H.c. }
\end{aligned}
$$

gives the laser coupling, where $\Omega_{j}$ denote the Rabi frequencies and $k_{j}$ the corresponding laser wave vectors $(j$ $=B, R, C)$. The relaxation operator

$$
\begin{aligned}
\mathcal{L} \rho= & -\frac{1}{2} \gamma_{P}(\rho|P\rangle\langle P|+| P\rangle\langle P| \rho)+\beta_{P S} \gamma_{P}|S\rangle\langle P|\rho| P\rangle\langle S| \\
& +\beta_{P D} \gamma_{P}|D\rangle\langle P|\rho| P\rangle\langle D|
\end{aligned}
$$

describes the radiative processes, coupling $|P\rangle$ to states $|S\rangle$ and $|D\rangle$, with decary rate $\gamma_{P} \simeq 20 \mathrm{MHz}$ and branching ratio $\beta_{P S} / \beta_{P D} \simeq 15$ for $\mathrm{Ca}^{+}$and $\beta_{P S}+\beta_{P D}=1$.

The radiative decay of state $|Q\rangle$, whose lifetime is about $1 \mathrm{~s}$ for $\mathrm{Ca}^{+}$, will be neglected in the analytical model we present below, but it is taken into account in the numerical calculations. It should also be noted that there is a large difference, by some orders of magnitude, between the dipole and the quadrupole couplings. In this manuscript we will focus on situations in which state $|Q\rangle$ is weakly coupled to the $\Lambda$ scheme, and which are thus experimentally feasible with standard laser sources. Finally, we will make reference to the dynamics of a single ion, as it can be realized in radio-frequency traps, and we will characterize its response by means of the occupation probabilities of the atomic levels, which can be monitored by resonance fluorescence or electron shelving techniques [25]. We will also take into account the ion's oscillatory motion in the trap.

\section{INTERNAL DYNAMICS OF A LOCALIZED PARTICLE}

In this section we focus on the solutions of Eq. (1) when the motion of the particle can be neglected, i.e., for steep traps and efficient cooling, such that the amplitude of its residual oscillations is much smaller than the laser wavelength. We study the dynamics with simple analytical models and compare their predictions with the results obtained from numerically solving the optical Bloch equations, derived from Eq. (1). We analyze the steady state and the time evolution under two particular conditions (i) the three-photon resonance case

$$
\Delta_{B}-\Delta_{R}-\Delta_{C}=0 \text { and } \Delta_{C} \neq 0
$$

in which states $|D\rangle$ and $|Q\rangle$ are resonantly coupled by threephoton processes and (ii) the $(2+1)$-photon resonance case

$$
\Delta_{R}-\Delta_{B}=0 \text { and } \Delta_{C}=0
$$

in which states $|S\rangle$ and $|D\rangle$ are coupled resonantly by a twophoton transition while $|S\rangle$ and $|Q\rangle$ are coupled resonantly by a one-photon process.

The major difference between the two cases is that when the $(2+1)$-photon resonance condition (ii) is fulfilled, the steady state response of the $\Lambda$-system alone would be characterized by a dark resonance, or coherent population trapping, resulting from the destructive interference between the 
two excitation paths $|S\rangle \rightarrow|P\rangle$ and $|D\rangle \rightarrow|P\rangle$ [9]. This has profound consequences also for the four-level dynamics, as will be shown below.

\section{A. Dressed states analysis}

In order to get some insight, we evaluate the dressed states of the system in the two limiting cases. We first focus on the three-photon resonance, condition (5), in the situation when the coupling between states $|Q\rangle$ and $|S\rangle$ can be treated perturbatively. We hence assume $\Delta_{C} \neq 0$ and $\Omega_{C} \ll\left|\Delta_{C}\right|$. The coupling between $|Q\rangle$ and $|S\rangle$ is in first order in the perturbation parameter $\alpha_{C}=\Omega_{C} / 2 \Delta_{C}$, and corrections to the states $|Q\rangle$ and $|S\rangle$ are at second order in $\alpha_{C}$, according to

$$
\begin{aligned}
& \left|S_{Q}\right\rangle=\mathcal{N}\left(|S\rangle+\alpha_{C}|Q\rangle\right), \\
& \left|Q_{S}\right\rangle=\mathcal{N}\left(|Q\rangle-\alpha_{C}|S\rangle\right),
\end{aligned}
$$

where $\mathcal{N}$ gives the correct normalization. The eigenfrequencies for these two states are $\alpha_{C} \Omega_{C} / 2$ and $-\Delta_{C}-\alpha_{C} \Omega_{C} / 2$, as displayed in Fig. 2(a). Here, $|D\rangle$ and $\left|Q_{S}\right\rangle$ are resonantly coupled by an effective two-photon process, and the system can be pumped into the eigenstate

$$
\left|\Psi_{N C}\right\rangle=\mathcal{N}^{\prime}\left(\mathcal{E}|D\rangle+\left|Q_{S}\right\rangle\right)
$$

with

$$
\mathcal{E}=\frac{\Omega_{B}}{\Omega_{R}} \alpha_{C}
$$

and normalization factor $\mathcal{N}^{\prime}$. This state is stable at second order in $\alpha_{C}$, it has the property of a dark state which is occupied asymptotically, thus signalling coherent population trapping [9]. According to this description, the corresponding electronic occupations at steady state $\mathcal{P}_{j}^{(N C)}=\left|\left\langle j \mid \Psi_{N C}\right\rangle\right|^{2}(j$ $=Q, D, S, P)$, are

$$
\begin{gathered}
\mathcal{P}_{Q}^{(N C)}=\frac{1}{1+\alpha_{C}^{2}+\mathcal{E}^{2}}+\mathrm{O}\left(\alpha_{C}^{4}, \mathcal{E}^{4}\right), \\
\mathcal{P}_{D}^{(N C)}=\frac{\mathcal{E}^{2}}{1+\mathcal{E}^{2}}+\mathrm{O}\left(\alpha_{C}^{4}, \mathcal{E}^{4}\right), \\
\mathcal{P}_{S}^{(N C)}=\frac{\alpha_{C}^{2}}{1+\alpha_{C}^{2}+\mathcal{E}^{2}}+\mathrm{O}\left(\alpha_{C}^{4}, \mathcal{E}^{4}\right),
\end{gathered}
$$

while $\mathcal{P}_{P}^{(N C)}=\mathrm{O}\left(\alpha_{C}^{4}\right)$. Hence, the parameter $\mathcal{E}$, or more precisely the ratio $\Omega_{B} / \Omega_{R}$ compared to $1 / \alpha_{C}$, determines the distribution of population between states $|Q\rangle$ and $|D\rangle$. A typical experimental situation is that $\Omega_{B}$ and $\Omega_{R}$ are similar, such that we concentrate on the case $\Omega_{B} / \Omega_{R} \ll 1 / \alpha_{C}$, i.e., $\mathcal{E} \ll 1$, and we see that the atom will occupy $|Q\rangle$ with almost unit probability. In this regime, the linewidth of state $|Q\rangle$ is due to higher-order coupling in $\alpha_{C}$ to state $|P\rangle$, and scales with $\left(\alpha_{C}^{2} \Omega_{B} / \Omega_{R}\right)^{2}$.

We now consider the case in which the 2+1-photon resonance condition (6) is fulfilled. We still restrict the discussion to the regime in which $\Omega_{C}$ is weak compared to all other
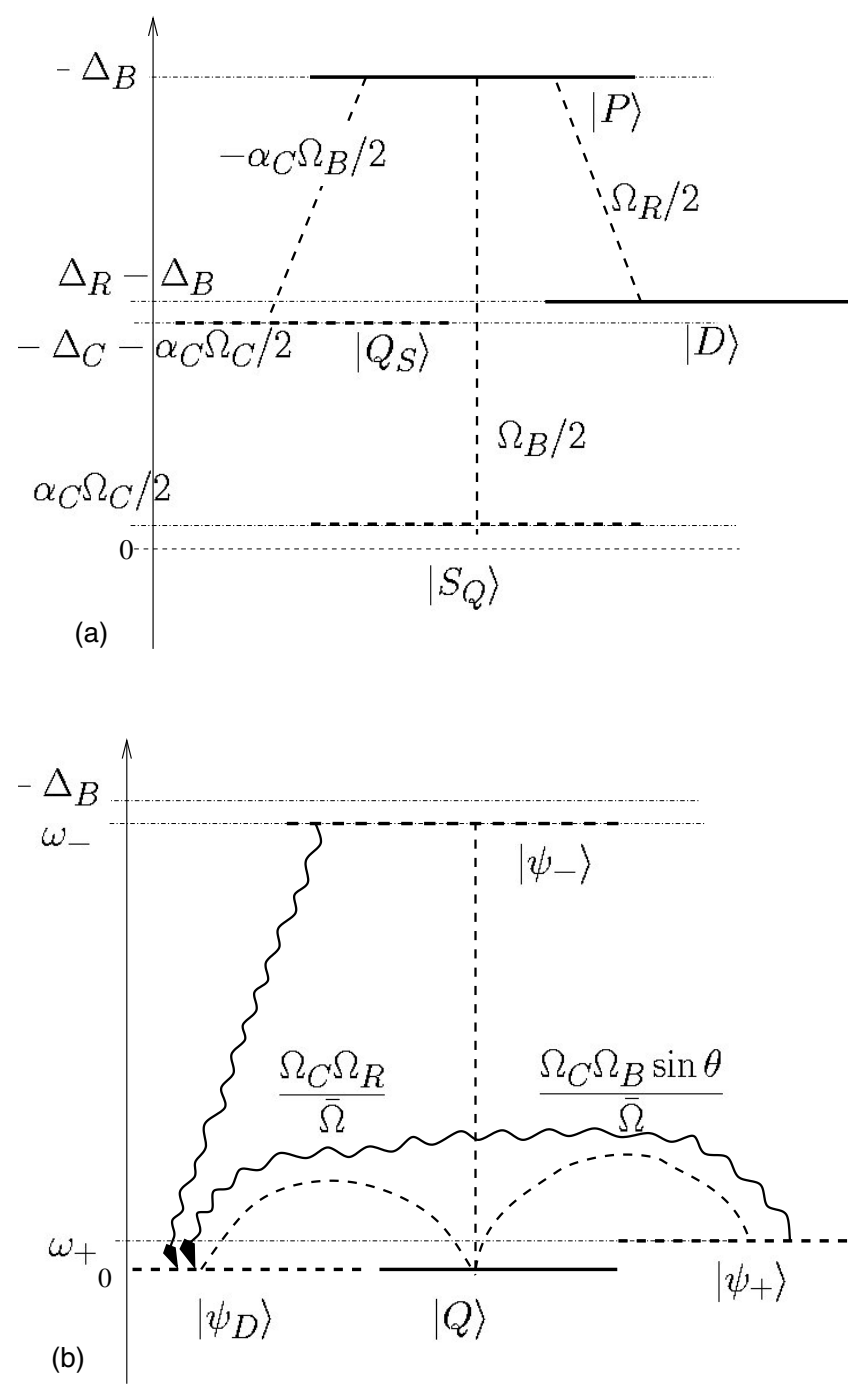

FIG. 2. Dressed states picture for (a) the three-photon resonance case and (b) the $(2+1)$-photon resonance case. See text for parameters and definitions.

coupling terms. In this case it is convenient to consider the eigenstates of the $\Lambda$ system formed by $\{|S\rangle,|P\rangle,|D\rangle\}$,

$$
\begin{gathered}
\left|\psi_{D}\right\rangle=\left(\Omega_{R}|S\rangle-\Omega_{B}|D\rangle\right) / \bar{\Omega}, \\
\left|\psi_{+}\right\rangle=\cos \theta|P\rangle+\sin \theta\left(\Omega_{B}|S\rangle+\Omega_{R}|D\rangle\right) / \bar{\Omega}, \\
\left|\psi_{-}\right\rangle=-\sin \theta|P\rangle+\cos \theta\left(\Omega_{B}|S\rangle+\Omega_{R}|D\rangle\right) / \bar{\Omega}
\end{gathered}
$$

with $\bar{\Omega}=\sqrt{\Omega_{R}^{2}+\Omega_{B}^{2}}$ and $\tan \theta=\left(\Delta_{B}+\sqrt{\Delta_{B}^{2}+\bar{\Omega}^{2}}\right) / \bar{\Omega}$ with $0 \leqslant \theta$ $<\pi / 2$. The dressed states of the diagonalized $\Lambda$ system are at frequencies

$$
\omega_{D}=0
$$

and 

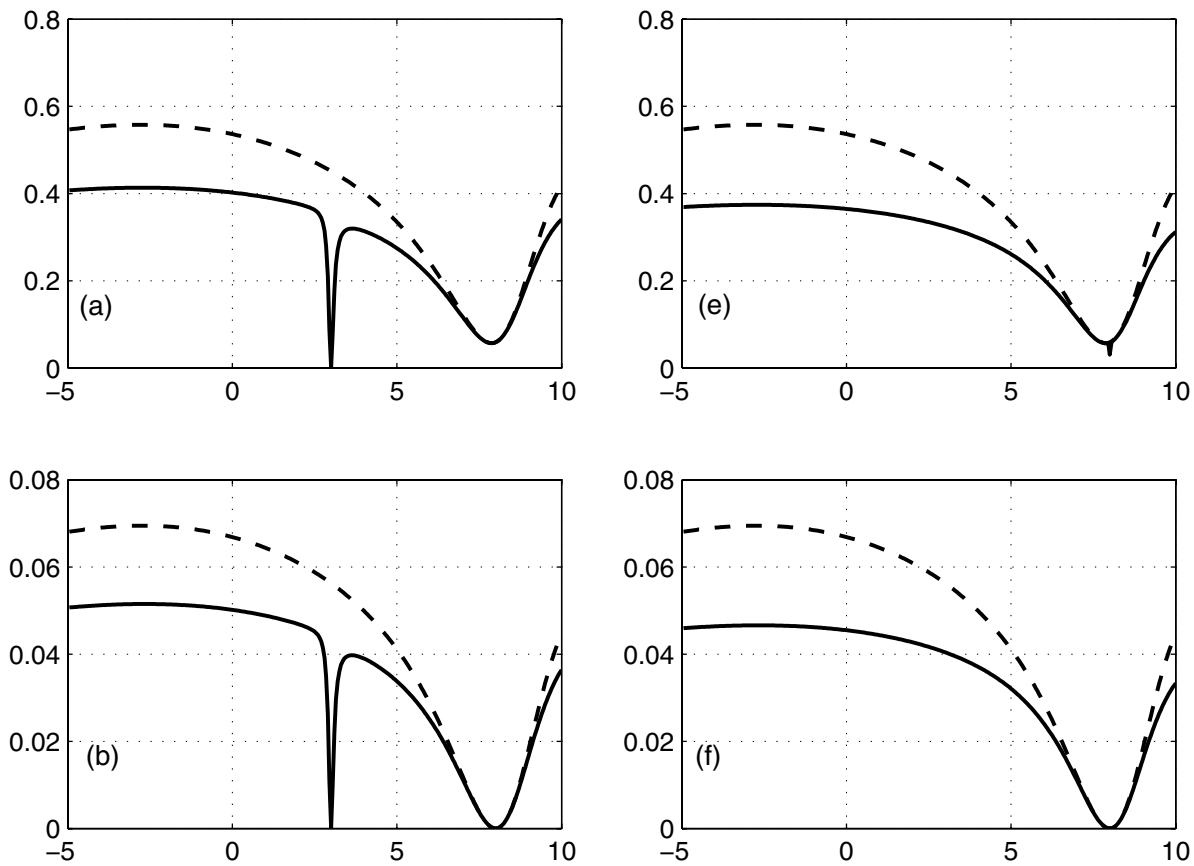

FIG. 3. Steady state populations $\mathcal{P}_{j}$ as a function of the detuning $\Delta_{R} / 2 \pi$. Displayed are $\mathcal{P}_{S}, \mathcal{P}_{P}$, $\mathcal{P}_{D}$, and $\mathcal{P}_{Q}$, from top to bottom. The parameters are $\Delta_{B}=2 \pi$ $\times 8 \mathrm{MHz}, \quad \Omega_{C}=2 \pi \times 0.05 \mathrm{MHz}$, $\Omega_{B}=2 \pi \times 10 \mathrm{MHz}$, and $\Omega_{R}=2 \pi$ $\times 2.5 \mathrm{MHz}$. In (a)-(d) $\Delta_{C}=2 \pi$ $\times 5 \mathrm{MHz}$, such that the three-
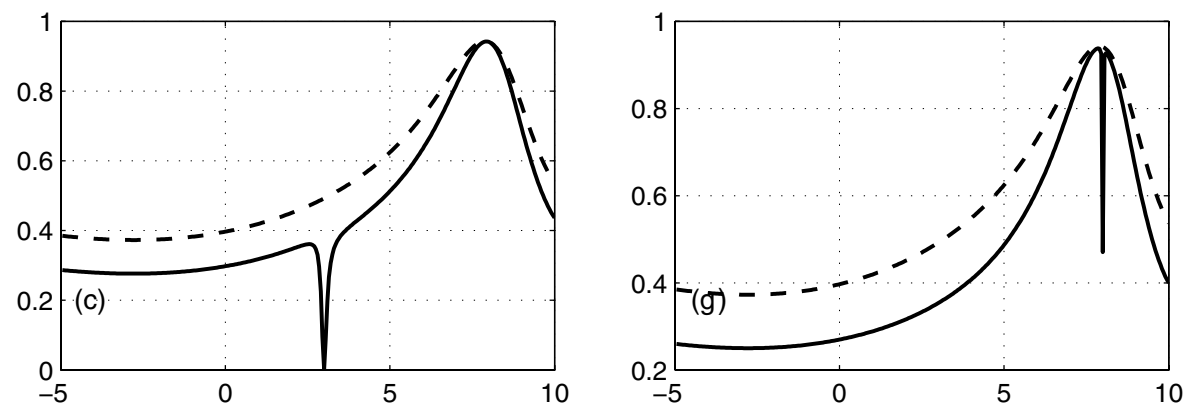
photon resonance is fulfilled at $\Delta_{R}=2 \pi \times 3 \mathrm{MHz}$; in (e)-(g) $\Delta_{C}$ $=0$, such that the $(2+1)$-photon resonance is found at $\Delta_{R}=2 \pi$ $\times 8 \mathrm{MHz}$. The dashed lines give the steady state populations for the same parameters but no coupling to $|Q\rangle\left(\Omega_{C}=0\right)$.
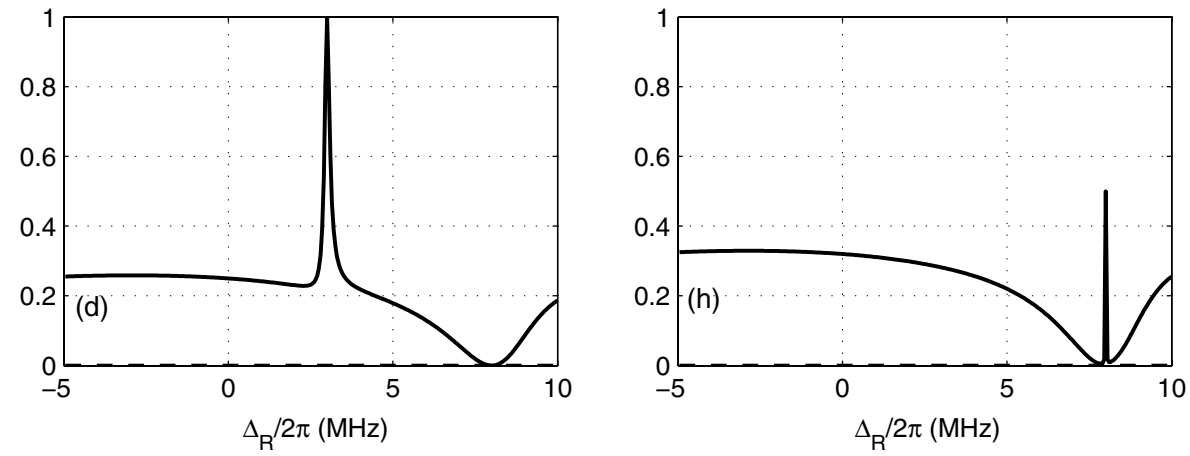

$$
\omega_{ \pm}=-\frac{1}{2}\left(\Delta_{B} \mp \sqrt{\Delta_{B}^{2}+\bar{\Omega}^{2}}\right) .
$$

The level scheme in this basis is sketched in Fig. 2(b). Here, $\left|\psi_{D}\right\rangle$ is the dark state of the $\Lambda$ system [9]. At $(2+1)$-photon resonance, state $|Q\rangle$ is resonantly coupled to the dark state $\left|\psi_{D}\right\rangle$ at rate $\widetilde{\Omega}_{C}=\Omega_{C} \Omega_{R} / \bar{\Omega}$, and for sufficiently weak coupling, $\Omega_{C} \ll\left|\omega_{ \pm}\right|$, there will be a time scale on which the dynamics of the system can be reduced to resonant two-level dynamics between these two states. For longer times, offresonant coupling between $|Q\rangle$ and $\left|\omega_{ \pm}\right\rangle$gives rise to damp- ing, and the system approaches the steady state at a rate which scales with the ratio $\Omega_{B}^{2} / \bar{\Omega}^{2}$. This damping gives rise also to the small residual linewidth of the $(2+1)$-photon resonance in the spectra shown in the next section.

\section{B. Steady state}

In order to illustrate the spectroscopic significance of the dressed states, we now discuss the steady-state populations $\mathcal{P}_{j}$ of the electronic levels $(j=S, P, D, Q)$ as a function of $\Delta_{R}$, for the two cases $\Delta_{C} \neq 0$ and $\Delta_{C}=0$, see Fig. 3. For highlighting the peculiarities of the four-level dynamics, we compare 

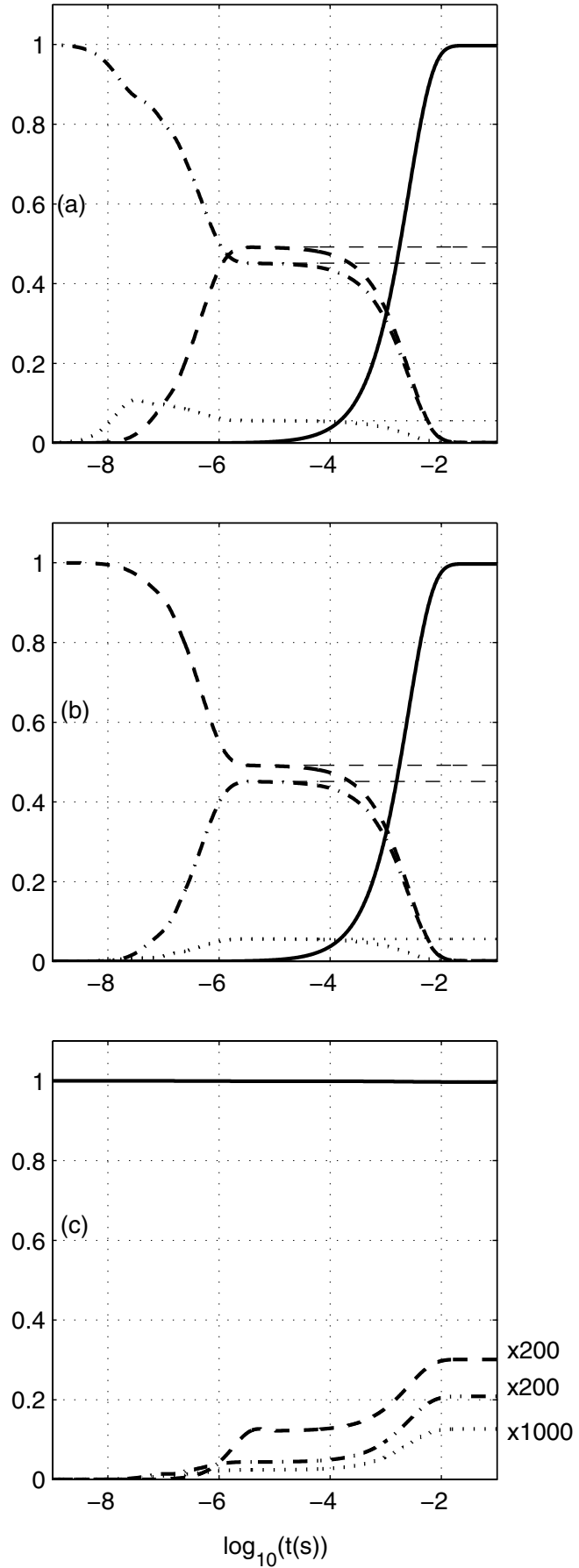
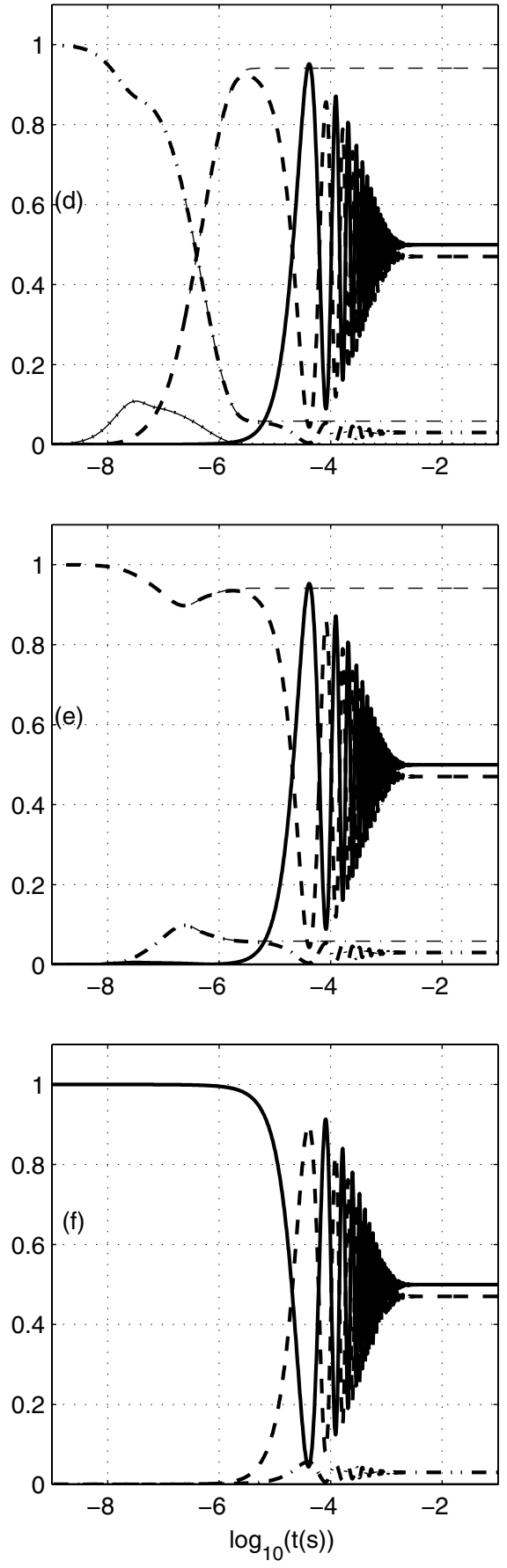

FIG. 4. Time evolution of the atomic level populations at three(left) and $(2+1)$-photon resonance (right), for different initial states. The lines correspond to the $|Q\rangle$ state (solid), $|D\rangle$ state (dashed), $|S\rangle$ state (dash-dotted), and $|P\rangle$ state (dotted). The initial state is $|S\rangle$ in (a), (d), $|D\rangle$ in (b), (e), and $|Q\rangle$, in (c), (f). Parameters in (a)(c) are the same as in Figs. 3(a)-3(d), and in (d)-(f) the same as in Figs. 3(e)-3(h). The thinner curves give the result in absence of coupling to state $|Q\rangle\left(\Omega_{C}=0\right)$, plotted on a logarithmic time scale. them to the behavior of the unperturbed $\Lambda$ system $\left(\Omega_{C}=0\right)$, whose stationary level occupations are indicated by the dashed curves; they exhibit the dark resonance at $\Delta_{R}=\Delta_{B}$, corresponding to suppressed population of $|P\rangle$. The effect of the weak coupling to state $|Q\rangle$ is represented by the solid curves.

Figures 3(a)-3(d) display the case $\Delta_{C} \neq 0$. One observes that coupling to $|Q\rangle$ does not change the behavior around the dark resonance, but it induces a critical change when $\Delta_{R}$ is at three-photon resonance: at this value all population is transferred to state $|Q\rangle$, Fig. 3(d), while all other states are correspondingly emptied. The width of this resonance is controlled by the ratio $\Omega_{R} / \Omega_{B}$, as discussed in the previous section. With the parameters chosen in Fig. $3\left(\Omega_{R} / \Omega_{B}\right.$ $=0.25$ ), the resonance is narrow but it can be made broader by increasing this ratio. In general, occupation of state $|Q\rangle$ at three-photon resonance is controlled by the parameter $\mathcal{E}$, as has been pointed out in the dressed state picture in Sec. III A. The important finding in this context is that in the regime $\mathcal{E} \ll 1, \mathcal{P}_{Q}$ is very close to unity, independent of the value of $\Omega_{C}$. This is only limited for very small values of the coupling by the decay of level $|Q\rangle$. In practical terms, this allows for robust preparation of the atom in $|Q\rangle$, by tuning the lasers to the three-photon resonance.

Figures 3(e)-3(h) display the stationary populations as a function of $\Delta_{R}$ when $\Delta_{C}=0$. At $(2+1)$-photon resonance, the 
coupling to $|Q\rangle$ gives rise to a transfer of $50 \%$ of the atomic population from the dark state of the $\Lambda$ system to $|Q\rangle$. In contrast to the case of $\Delta_{C} \neq 0$, this is indeed the maximum occupation that $|Q\rangle$ can achieve for $\Delta_{C}=0$. This is understood considering the dressed state picture in Sec. III A: for $\Delta_{C}=0$ dark state $\left|\psi_{D}\right\rangle$ and $|Q\rangle$ form a resonantly coupled two-level system, where damping is weak and arises only from off-resonant coupling. Hence, at steady state the populations of the two states are the stationary populations of a saturated dipole.

The two examples reported here show that a weak coupling to a fourth state which is metastable can change dramatically the response of a $\Lambda$ system when the detunings fulfill, or are around, the three-photon resonance condition. Three-photon processes were previously studied in Refs. $[19,20]$ in an atomic model system where decay of state $|P\rangle$ into $|Q\rangle$ is allowed. This constitutes a major difference to the dynamics discussed here: in the model of Refs. $[19,20]$ one does not observe the narrow three-photon resonance for weak coupling $\Omega_{C}$, as population is optically pumped into $|Q\rangle$ for a wide range of values of $\Delta_{R}$.

\section{Time evolution at three-photon resonance}

Let us now consider how the atomic level occupation evolves as a function of time. Figures 4(a)-4(c) display the time evolution for different initial conditions when the atom is driven at three-photon resonance and when $\left|\Delta_{C}\right| \gg \Omega_{C}$, i.e., under conditions for which the atom is found in $|Q\rangle$ at steady state. The thinner curves are plotted for comparison and indicate the corresponding three-level dynamics, evaluated by setting $\Omega_{C}=0$. When the initial state is $|S\rangle$ or $|D\rangle$ [Figs. 4(a) and 4(b)], one can identify a clear hierarchy of couplings: on a short time scale, within about $1 \mu$ s for the chosen parameters, the system evolves to the steady state of the $\Lambda$ configuration. On a longer time scale $(\sim 1 \mathrm{~ms})$, population is transferred to state $|Q\rangle$ through its coupling to the $\Lambda$ scheme. When the system has been prepared in state $|Q\rangle$ [Fig. 4(c)], it essentially remains in that state during all time, apart from a small redistribution of population from $|Q\rangle$ to $|D\rangle$ on the slow time scale.

Such a dynamical behavior indicates the appearance of quantum jumps [26-28], i.e., of randomly alternating phases of full and no fluorescence: after the emission of a photon, which projects the atom into either $|S\rangle$ or $|D\rangle$, the atom quickly assumes a quasisteady state (the steady state of the $\Lambda$ system) which has significant population in $|P\rangle$ and is therefore likely to scatter more photons. The average duration of these bright periods is given by the slow time scale on which the system evolves into $|Q\rangle$. With the parameters of this example, an average of about $3 \times 10^{3}$ photons are scattered during a bright period. When the system has made a transition to $|Q\rangle$, signalled by a dark time much longer than the typical interval between two scattered photons [29], then it will remain there for the average duration of the dark periods, i.e., the long time scale on which $|Q\rangle$ couples to the remaining three states [Fig. 4(c)].

Figures 4(d)-4(f) display the time evolution out of various initial states, when the $(2+1)$-photon resonance is ful-

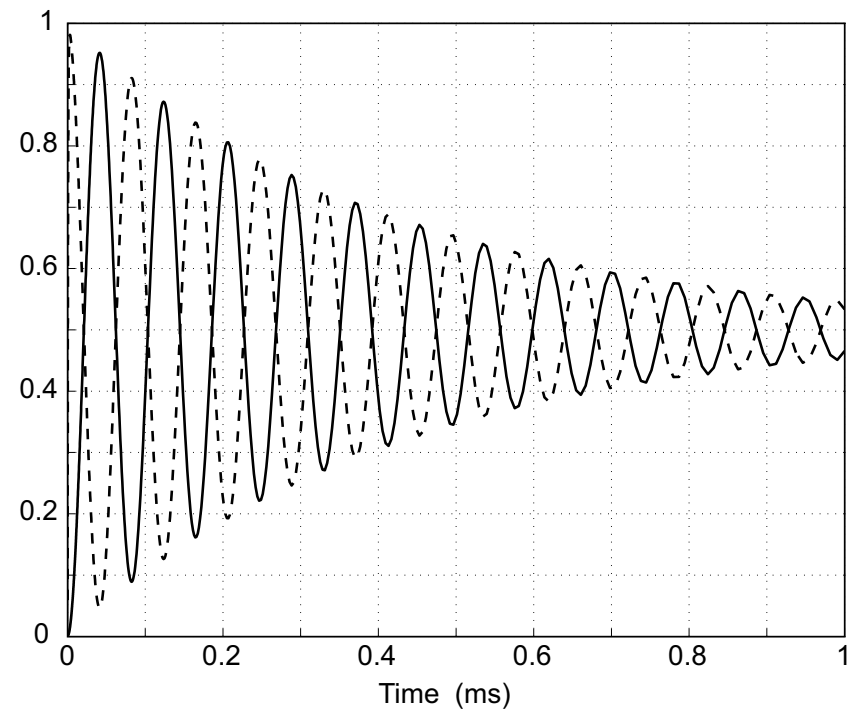

FIG. 5. Rabi oscillations between states $\left|\psi_{D}\right\rangle$ (dashed line) and $|Q\rangle$ (solid line), corresponding to Fig. 4(d) but plotted on a linear time scale.

filled. The dynamics are again well separated into different time scales: the system accesses very quickly (within $\sim 1 \mu \mathrm{s}$ ) the steady state of the $\Lambda$ system, which in this case is the dark state of Eq. (13) with no population in $|P\rangle$. On the very long time scale $(>1 \mathrm{~ms})$, the global steady state including $|Q\rangle$ is assumed. The important observation, peculiar for this $(2+1)$-photon case, are oscillations on the intermediate time scale, between the populations of $|D\rangle,|S\rangle$, and $|Q\rangle$, whereby $|D\rangle$ and $|S\rangle$ oscillate in phase with each other, and in antiphase with $|Q\rangle$. This is consistent with the dressed state analysis of Sec. III A and corresponds to Rabi oscillations between the dark state $\left|\psi_{D}\right\rangle$ and the state $|Q\rangle$. Figure 5 highlights these oscillations, whose frequency is determined by the effective Rabi coupling $\Omega_{C} \Omega_{R} / \bar{\Omega}$. Hence, the overall dynamics of the $(2+1)$-photon resonance case are characterized by an initial dissipative behavior which evolves into a period of coherent dynamics, i.e., Rabi oscillations between $\left|\psi_{D}\right\rangle$ and $|Q\rangle$; finally, these Rabi oscillations are also damped out through off-resonant coupling to states $\left|\psi_{ \pm}\right\rangle$, which decay incoherently.

We remark that in this situation a single atom would be observed to switch spontaneously between incoherent and coherent dynamics: as the damping of the Rabi oscillations corresponds to the emission of a photon, it will trigger the dynamics of Fig. 4(d) to start again. This type of quantumjump behavior should be revealed in an experiment through long-time oscillations of the $g^{(2)}(\tau)$ photon-photon correlation function.

Moreover, the coherent coupling between the dark and the $|Q\rangle$ state can be brought to an extreme behavior: in the limit $\Omega_{R} \ll \Omega_{B}$ the dark state practically coincides with state $|D\rangle$, and one observes Rabi oscillations between states $|D\rangle$ and $|Q\rangle$, where population is directly and coherently transferred between the two states by means of three-photon processes.

\section{EFFECTS OF THE CENTER-OF-MASS MOTION}

So far we have neglected the effect of the center-of-mass motion on the atomic dynamics. The motion may however 

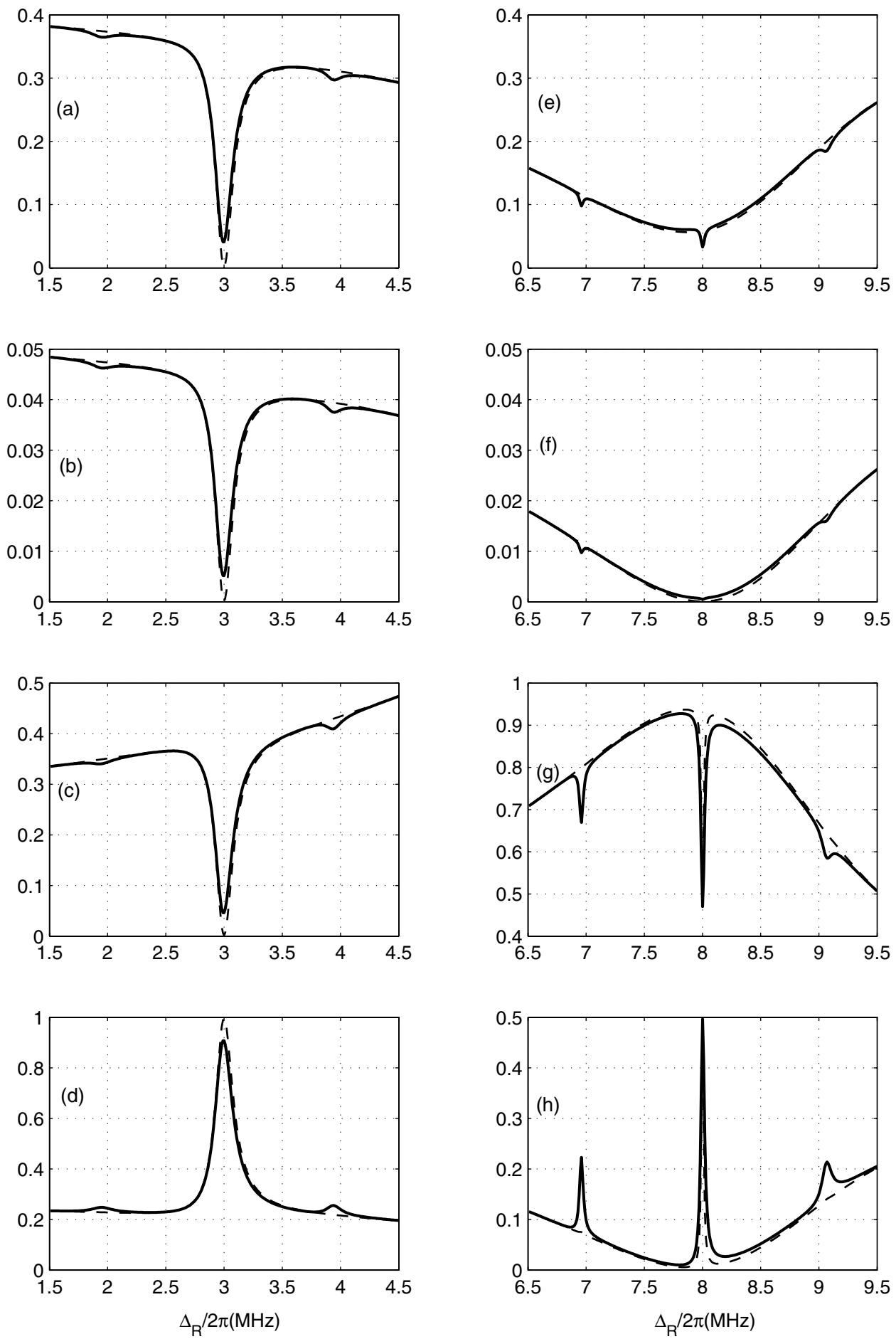

FIG. 6. Steady state populations $\mathcal{P}_{S}, \mathcal{P}_{P}, \mathcal{P}_{D}$, and $\mathcal{P}_{Q}$ (from top to bottom) versus detuning $\Delta_{R} / 2 \pi$ for the same parameters as in Fig. 3 and taking into account the oscillation of the $\mathrm{Ca}^{+}$ion in a trap at frequency $\nu=2 \pi \times 1 \mathrm{MHz}$ and Lamb-Dicke parameters $\left|\eta_{B, R, C}\right|$ $=(0.1,0.046,0.054)$. The dashed line corresponds to the case of copropagating lasers (minimal $\left.\Delta \vec{k}=\vec{k}_{B} \times 0.003\right)$, the solid line to $\vec{k}_{R}$ and $\vec{k}_{C}$ copropagating against $\vec{k}_{B}$ (maximal $\Delta \vec{k} \simeq \vec{k}_{B} \times 2$ ). The left column illustrates the three-photon resonance, the right one the $(2+1)$-photon resonance.

critically affect the atomic response, and the $N$-type level scheme we are considering has been subject of several studies of how inhomogeneous broadening affects light transmission in atomic vapors [20-22]. Let us start with some general considerations for our particular system. In the dressed state picture at three-photon resonance [condition (5)], motion of the atom at momentum $\vec{p}$ gives rise to a Doppler effect which lifts the degeneracy between states $|D\rangle$ and $|Q\rangle$. It thus gives rise to an instability of state $\left|\Psi_{N C}\right\rangle$, Eq. (9), which now couples to state $\left|\Psi_{C}\right\rangle=\mathcal{N}^{\prime}\left(|D\rangle-\mathcal{E}\left|Q_{S}\right\rangle\right)$ at rate 

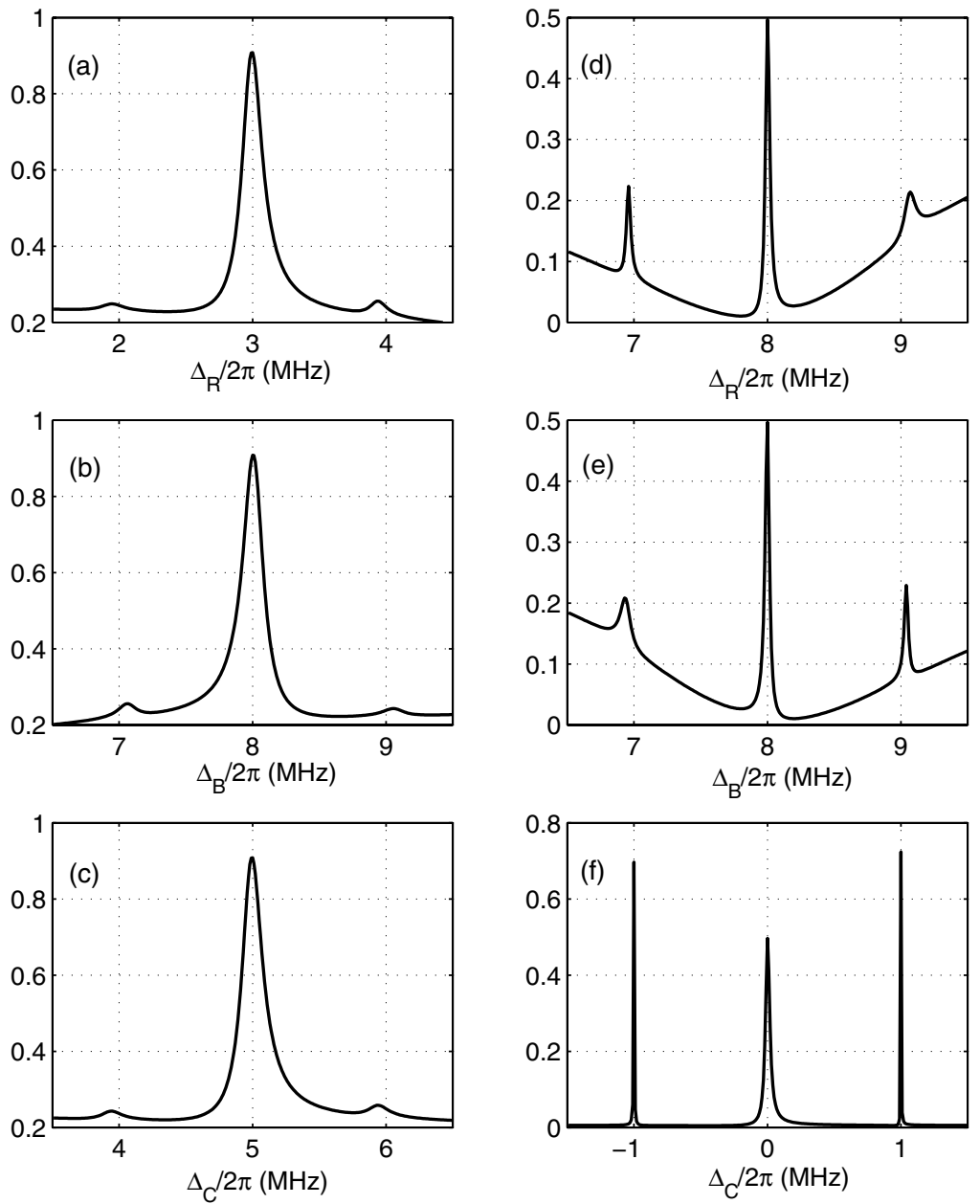

FIG. 7. Steady state population $\mathcal{P}_{Q}$ versus detunings $\Delta_{R} / 2 \pi, \Delta_{B} / 2 \pi$, and $\Delta_{C} / 2 \pi$ (from top to bottom), for the same parameters as in Fig. 6, and for $\vec{k}_{R}$ and $\vec{k}_{C}$ copropagating against $\vec{k}_{B}\left(\Delta \vec{k} \simeq \vec{k}_{B}\right.$ $\times 2)$. The left column illustrates the three-photon resonance, the right one the $(2+1)$-photon resonance.

$$
\mathcal{R}=\left\langle\Psi_{N C}|H| \Psi_{C}\right\rangle \approx \mathcal{E} \frac{\vec{p}}{m} \cdot\left(\vec{k}_{R}-\vec{k}_{B}+\vec{k}_{C}\right)
$$

This coupling may sensitively affect the dynamics of the system, due to the narrow resonance condition. It vanishes, however, in the geometric Doppler-free three-photon resonance condition (phase-matching condition)

$$
\Delta \vec{k}=\vec{k}_{R}-\vec{k}_{B}+\vec{k}_{C}=\overrightarrow{0}
$$

This configuration has been studied in Refs. [20,22]. In addition, in the same configuration but for $\Delta \vec{k} \neq \overrightarrow{0}$, Dopplerinsensitive three-photon resonances have been observed when $|S\rangle \rightarrow|Q\rangle$ is in the radio-frequency regime [22]. In this special case, the radio-frequency coupling gives rise to sidebands and thus to a discrete spectrum of excitations on the transition $|S\rangle \rightarrow|Q\rangle$ which couple quasiresonantly to $|D\rangle$ for different velocity classes [20].

In our model system, ${ }^{40} \mathrm{Ca}^{+}$, all transitions are in the optical regime. We account for the oscillatory motion of the ion inside the trapping potential by a time-dependent position

$$
\vec{x}(t)=\vec{x}_{0} \cos \nu t,
$$

where $\vec{x}_{0}$ is the classical oscillation amplitude and $\nu$ the frequency of oscillation [30]. In the Hamiltonian (3), the effect is a modulation of the radiative coupling. In this manuscript we will assume the Lamb-Dicke regime, using the LambDicke parameters $\eta_{j}=\vec{k}_{j} \cdot \vec{x}_{0} / 2(j=S, P, D, Q)$ as small perturbative parameters. We then use a Floquet ansatz for studying the stationary response of the system. The basic equations are reported in the Appendix.

Figure 6 shows the steady state populations of the four electronic levels around the three-photon resonance (left column) and the $(2+1)$-photon resonance (right column) for different laser beam geometries. The dashed curves correspond to the case where the lasers are copropagating, which for $\mathrm{Ca}^{+}$ gives rise to a small three-photon Doppler effect: the two metastable states have very close energy levels, and in this configuration, the effective wave vector is $\Delta \vec{k}=\vec{k}_{B} \times 0.003$. We see that, in this case, the effect of the motion does not change appreciably the steady state occupation.

The solid lines in Fig. 6 correspond to a Doppler sensitive geometry, where $\vec{k}_{B}$ counter-propagates against $\vec{k}_{R}$ and $\vec{k}_{C}$. For this geometry one can observe sidebands in the resonance profile. Moreover, there are major differences between the three-photon and the $(2+1)$-photon resonance cases, in which the sideband signals are significantly narrower and higher. One also observes that in the three-photon resonance case the height of the central band of $\mathcal{P}_{Q}$ is reduced by the effect of the motion to a value smaller than unity [Fig. 6(d)] while for the $(2+1)$-photon resonance the central band of $\mathcal{P}_{Q}$ still reaches the maximum value $1 / 2$. Further insight can be 
gained by comparing the spectra obtained by scanning the three different lasers, as shown in Fig. 7. One clearly observes that the sidebands are especially high when they fall in the dark-resonance region, and that they may be larger than the central band when the $(2+1)$-resonance condition is fulfilled between the dark state and one of the sidebands [Fig. 7(f)]. In this latter case, they achieve a value larger than $1 / 2$ due to the contribution of three-photon processes which include one sideband transition.

\section{DISCUSSION AND CONCLUSIONS}

The weak coupling of a $\Lambda$-shaped three-level system to a fourth metastable state modifies critically the dynamics at three-photon resonance. In a wide regime of parameters, the metastable level is occupied with unit probability at steady state. The time evolution shows that the dynamics of the system is characterized by two time scales, a short one corresponding to the dynamics of the $\Lambda$ system alone, and a longer one corresponding to its coupling to the metastable state: on this time scale population is transferred into it. When the $\Lambda$ system is driven at two-photon resonance and the three-photon resonance condition is fulfilled, the system first accesses the three-level dark state through dissipation and then switches to coherent dynamics, characterized by Rabi oscillations between dark and metastable state. By choosing the coupling properly, these dynamics can reduce to direct coherent transfer (Rabi oscillations) between the two extremal states coupled at three-photon resonance.

In general, the center-of-mass motion modifies substantially the response of the system, due to the sensitivity of the narrow resonances to Doppler shifts. The narrow threephoton resonance is recovered in configurations of the laser beams for which the Doppler effect on the three-photon transition is suppressed. In this manuscript we have considered the effect of the oscillatory motion of a tightly trapped atom, and we have observed that the motional sidebands which appear in the spectra can be significantly enhanced due to interfering two- and three-photon processes.

In an experimental realization, finite laser bandwidths will be detrimental for the creation of quantum coherences. The three-photon resonance can be still observed by broadening its linewidth, increasing the ratio $\Omega_{B} / \Omega_{R}$. Numerical calculations show that more than $97 \%$ occupation of state $|Q\rangle$ can be reached with lasers of bandwidth $10 \mathrm{kHz}$ (HWHM) under the three-photon resonance condition. For the same parameters, the effect of the laser bandwidth at $(2+1)$-photon resonance leads to a reduction of the population by only $0.2 \%$ with respect to the ideal case.

In conclusion, we have shown that the weak perturbation of a $\Lambda$ system, achieved by coupling to a metastable state, gives rise to interesting dynamics. We have provided simple pictures for understanding them. These can find applications for high-precision measurement, for instance for metrology in the spirit of Refs. [8,24], quantum state preparation and manipulation as, for instance, in transistors for single atoms as in Ref. [7].

\section{ACKNOWLEDGMENTS}

C.C. thanks Jean Dalibard for very helpful and stimulating discussions. G.M. acknowledges discussions with Ramon Corbalán and the kind hospitality of the Laboratoire PIIMCIML at the Université de Provence under a visiting professor grant. This work was partly supported by the French Ministère des affaires étrangères (Picasso 09133XH), by the Spanish Ministerio de Educación y Ciencia (Acción Integrada, HU2004-0015; LACSMY Project No. FIS200405830; programa Ramon-y-Cajal; QLIQS Project No. FIS2005-08257), and by the European Commission (SCALA Integrated Project, Contract No. 015714).

\section{APPENDIX: OSCILLATORY MOTION OF THE TRAPPED ATOM}

In the Lamb-Dicke regime, we decompose

$$
H_{I}(t)=H_{I}^{(0)}+e^{i \nu t} H_{I}^{+}+e^{-i \nu t} H_{I}^{-},
$$

with

$$
\begin{gathered}
H_{I}^{(0)}=\frac{\hbar \Omega_{B}}{2}|P\rangle\left\langle S\left|+\frac{\hbar \Omega_{R}}{2}\right| P\right\rangle\left\langle D\left|+\frac{\hbar \Omega_{C}}{2}\right| Q\right\rangle\langle S|+\text { H.c. }, \\
H_{I}^{ \pm}=i \eta_{B} \frac{\hbar \Omega_{B}}{2}|P\rangle\left\langle S\left|+i \eta_{R} \frac{\hbar \Omega_{R}}{2}\right| P\right\rangle\left\langle D\left|+i \eta_{C} \frac{\hbar \Omega_{C}}{2}\right| Q\right\rangle\langle S|+\text { H.c. }
\end{gathered}
$$

The solution can be found using a Floquet ansatz for the density matrix, hence writing

$$
\rho=\sum_{n=-\infty}^{\infty} \rho^{(n)} e^{i n \nu t} .
$$

Substituting into the master equation, we find the coupled equations

$$
\begin{aligned}
\frac{\partial}{\partial t} \rho^{(n)}= & -i n \nu \rho^{(n)}-\frac{i}{\hbar}\left[H_{0}+H_{I}^{(0)}, \rho^{(n)}\right]-\frac{i}{\hbar}\left[H_{I}^{+}, \rho^{(n-1)}\right] \\
& -\frac{i}{\hbar}\left[H_{I}^{-}, \rho^{(n+1)}\right]+\mathcal{L} \rho^{(n)}
\end{aligned}
$$

which have been obtained by neglecting higher orders in the Lamb-Dicke expansion.
[1] D. A. Braje, V. Balic, S. Goda, G. Y. Yin, and S. E. Harris, Phys. Rev. Lett. 93, 183601 (2004).

[2] V. Balic, D. A. Braje, P. Kolchin, G. Y. Yin, and S. E. Harris, Phys. Rev. Lett. 94, 183601 (2005).
[3] M. D. Eisaman, L. Childress, A. André, F. Massou, A. S. Zibrov, and M. D. Lukin, Phys. Rev. Lett. 93, 233602 (2004).

[4] M. D. Lukin and A. Imamoglu, Phys. Rev. Lett. 84, 1419 (2000). 
[5] C. Ottaviani, D. Vitali, M. Artoni, F. Cataliotti, and P. Tombesi, Phys. Rev. Lett. 90, 197902 (2003).

[6] I. Friedler, D. Petrosyan, M. Fleischhauer, and G. Kurizki, Phys. Rev. A 72, 043803 (2005).

[7] A. Micheli, A. J. Daley, D. Jaksch, and P. Zoller, Phys. Rev. Lett. 93, 140408 (2004).

[8] R. Santra, E. Arimondo, T. Ido, C. Greene, and J. Ye, Phys. Rev. Lett. 94, 173002 (2005).

[9] E. Arimondo, in Progress in Optics, edited by E. Wolf (Elsevier Science, Amsterdam, 1996), Vol. XXXV, Chap. V, p. 258.

[10] G. S. Agarwal and W. Harshawardhan, Phys. Rev. Lett. 77, 1039 (1996).

[11] M. D. Lukin, P. R. Hemmer, and M. O. Scully, Adv. At., Mol., Opt. Phys. 42, 347 (2000).

[12] G. Morigi, S. Franke-Arnold, and G. L. Oppo, Phys. Rev. A 66, 053409 (2002).

[13] B. S. Ham and P. R. Hemmer, Phys. Rev. Lett. 84, 4080 (2000).

[14] M. D. Lukin, Rev. Mod. Phys. 75, 457 (2003).

[15] A. V. Taichenachev, A. M. Tumaikin, and V. I. Yudin, Phys. Rev. A 61, 011802(R) (1999).

[16] C. Goren, A. D. Wilson-Gordon, M. Rosenbluh, and H. Friedmann, Phys. Rev. A 67, 033807 (2003).

[17] C. Goren, A. D. Wilson-Gordon, M. Rosenbluh, and H. Friedmann, Phys. Rev. A 69, 053818 (2004).

[18] A. M. Akulshin, S. Barreiro, and A. Lezama, Phys. Rev. A 57,
2996 (1998).

[19] M. D. Lukin, S. F. Yelin, M. Fleischhauer, and M. O. Scully, Phys. Rev. A 60, 3225 (1999).

[20] S. F. Yelin, V. A. Sautenkov, M. M. Kash, G. R. Welch, and M. D. Lukin, Phys. Rev. A 68, 063801 (2003).

[21] C. Y. Ye, A. S. Zibrov, Y. V. Rostovtsev, and M. O. Scully, Phys. Rev. A 65, 043805 (2002).

[22] A. S. Zibrov, C. Y. Ye, Y. V. Rostovtsev, A. B. Matsko, and M. O. Scully, Phys. Rev. A 65, 043817 (2002).

[23] B. Appasamy, I. Siemers, Y. Stalgies, J. Eschner, R. Blatt, W. Neuhauser, and P. E. Toschek, Appl. Phys. B B60, 473 (1995).

[24] T. Hong, C. Cramer, W. Nagourney, and E. N. Fortson, Phys. Rev. Lett. 94, 050801 (2005).

[25] H. Dehmelt, Bull. Am. Phys. Soc. 20, 60 (1975).

[26] W. Nagourney, J. Sandberg, and H. Dehmelt, Phys. Rev. Lett. 56, 2797 (1986).

[27] T. Sauter, W. Neuhauser, R. Blatt, and P. E. Toschek, Phys. Rev. Lett. 57, 1696 (1986).

[28] J. C. Bergquist, R. G. Hulet, W. M. Itano, and D. J. Wineland, Phys. Rev. Lett. 57, 1699 (1986).

[29] G. Nienhuis, Phys. Rev. A 35, 4639 (1987).

[30] Both the driven (micro) and the secular (macro) motion of a trapped ion may be described this way; unless quantum effects in the macro-motion are relevant, the thermal state of a laser cooled ion is modeled by additional integration over a thermal distribution of oscillation amplitudes. 